${ }^{+}+{ }^{+}{ }^{+}$La revue pour l'histoire du CNRS

POUR L'HISTOIRE DU CNRS $\quad 24 \mid 2009$

Soixante-dixième anniversaire du CNRS

\title{
Climat et avenir
}

Amy Dahan

\section{OpenEdition}

Journals

Édition électronique

URL : https://journals.openedition.org/histoire-cnrs/9055

DOI : 10.4000/histoire-cnrs.9055

ISSN : 1955-2408

Éditeur

CNRS Éditions

Édition imprimée

Date de publication : 5 octobre 2009

ISSN : 1298-9800

Référence électronique

Amy Dahan, «Climat et avenir », La revue pour l'histoire du CNRS [En ligne], 24 | 2009, mis en ligne le 05 octobre 2009, consulté le 20 mai 2021. URL : http://journals.openedition.org/histoire-cnrs/9055 ; DOI : https://doi.org/10.4000/histoire-cnrs.9055

Ce document a été généré automatiquement le 20 mai 2021.

Comité pour l'histoire du CNRS 


\section{Climat et avenir}

\section{Amy Dahan}

1 Avant la fin des années 1988, rien n'était clair en matière d'évolution du climat, la première identification de perturbations climatiques s'était faite avec la détection du trou dans la couche d'ozone dans les années 1970. Pour travailler sur le climat, les chercheurs ont besoin d'ordinateurs numériques extrêmement puissants pour pouvoir établir des modèles de référence. Aussi, l'évolution des recherches climatiques est-elle allée de pair avec les progrès technologiques des ordinateurs et des modes de calculs, et également avec la collecte des données grâce aux technologies spatiales.

2 Dès 1990, dans le cadre des Nations Unies, le GIEC a participé à divers processus, notamment le Sommet de la Terre à Rio en 1992. Si les Français ont fait une entrée timide dans ce GIEC, trois chercheurs ont joué un rôle important : Gérard Mégie, ancien président du CNRS, a créé l'institut Pierre-Simon- Laplace en 1991; Jean Jouzel', à l'origine spécialiste de la composition isotopique des grêlons, cofondateur du Laboratoire des sciences du climat et de l'environnement (CNRS-CEA); enfin, Hervé Le Treut $^{2}$, physicien spécialiste des modèles du climat.

3 Les premiers rapports du GIEC ont été très prudents dans leurs prédictions alors que certains climatologues étaient déjà persuadés de dangers beaucoup plus pressants. Ce n'est qu'avec le troisième rapport de cet organisme en 2001 que les choses se sont précisées. A. Dahan a pu, à travers une enquête, suivre la prise de conscience du danger par les scientifiques et leur engagement auprès du GIEC. En effet, selon la tradition scientifique, il est plus valorisant pour les chercheurs de faire de la recherche fondamentale que des simulations pour le GIEC : «Un modèle climatique est extrêmement compliqué à construire. Il faut faire de nombreuses simulations afin d'explorer et de comprendre chaque processus physique ou biochimique, ou alors pour avoir une fourchette de prédictions par rapport aux différents scénarios économiques pour la fin du XXIe siècle par exemple. Cette dernière tâche implique un long travail informatique et du temps de calcul au détriment d'autres recherches. Certains chercheurs préféraient travailler sur les mécanismes mêmes du climat. Pourtant, il a fallu faire les deux choses à la fois, c'est-à-dire continuer la recherche fondamentale et faire des simulations de scénarios pour le GIEC car les projections pour le futur ne fonctionnent qu'avec ces simulations ", souligne-t-elle. 
4 Les climatologues utilisent une large palette de modèles qui leur permettent de formuler des hypothèses, de chercher les causes des mécanismes et les tester, d'étudier le comportement d'une atmosphère simplifiée sous certaines conditions. Ces modèles autorisent la manipulation de climats virtuels, ou encore d'explorer le passé et le futur, etc.

5 En France, seuls deux organismes s'occupent de l'étude du climat: Météo France et l'institut Pierre- Simon-Laplace (qui fait partie de l'Institut national des sciences de l'Univers, Insu). Le CNRS est un véritable pilier de la recherche climatique. Initialement, le Laboratoire de météorologie dynamique (LMD) du CNRS n'a pas voulu utiliser les modèles climatiques de Météo France déjà performants en 1990. Préférant construire lui-même ses modèles, il a laissé pour cela une entière liberté aux chercheurs, ce qui a sans doute provoqué un petit retard. La création de l'institut Pierre-Simon-Laplace en 1991 sera une étape déterminante qui réunira des spécialistes des composantes du climat et toutes les compétences nécessaires. Gérard Mégie et Jean Jouzel y ont joué un rôle capital.

6 A. Dahan a voulu étudier les modèles climatiques. Réfléchissant à ces questions en tant qu'épistémologue et historienne des sciences, le climat lui est apparu comme un objet idéal pour saisir le fonctionnement des sciences contemporaines. Elle s'était déjà intéressée à divers modèles de la théorie du chaos, en physique avant de passer au problème des modèles de climat. D'emblée, elle coopère avec des climatologues, des économistes élaborant des modèles et scénarios économiques. Son intérêt se porte sur les questions épistémologiques dans la production des modèles et l'évolution des pratiques de modélisation; mais aussi sur les diverses communautés scientifiques engagées et les processus d'expertise dans ce domaine.

Dès 2002, ses études sur les modèles climatiques l'ont amenée à s'ouvrir à des questions a priori extérieures à son champ (politiques, économiques...). Selon elle, de 1988 à aujourd'hui, la question de la science du climat ne s'est pas développée sans être intimement liée aux questions politiques. Depuis 3- 4 ans, les États se demandent s'ils pourront appliquer ou non les mesures du protocole de Kyoto de 1997. On commence déjà à observer les changements climatiques qui s'opéreront dans le futur. Avec ces observations et les connaissances scientifiques révélées à l'espace public, des politiques en matière d'environnement et de développement durable doivent pouvoir s'imposer.

Pour A. Dahan, le changement climatique doit être l'occasion de remettre au-devant de la scène la question du développement durable parce que les réponses au défi climatique ont de grosses répercussions économiques, politiques et sociétales. Aussi, doit-on pouvoir redéfinir le contenu du développement durable de façon plus technique, plus concrète, plus précise et donner la possibilité à ces Géants du Sud tels que la Chine, le Brésil et l'Inde de faire des choix pour leur développement plus respectueux de l'environnement...

Il est désormais possible de réaliser des bilans carbones ${ }^{3}$ de toute activité économique, industrielle, etc. Si on construit une nouvelle usine, on peut utiliser cet indicateur plus simple que l'empreinte écologique. Cette unité de mesure est un élément important pour faire des comparaisons en matière agricole, technologique, etc. Amy Dahan s'est rendue compte depuis 2007 qu'un palier supplémentaire dans la prise de conscience avait été franchi, notamment dans la manière dont s'est tenue la conférence de Bali en décembre 2007, ou le retentissement qu'a eu l'attribution du prix Nobel de la paix ${ }^{4} 2007$ au GIEC. Son objectif est multiple: mieux comprendre le processus du changement 
climatique et élaborer des solutions respectueuses de l'individu et des sociétés afin de préserver le développement pour les générations futures...

\section{NOTES}

1.Jean Jouzel, ingénieur chimiste, directeur de cet Institut des sciences de l'environnement.

2. Hervé Le Treut, alors directeur de recherche au Laboratoire de météorologie dynamique du CNRS à l'École normale supérieure a écrit : « Pourquoi les modèles n'ont pas tort ", article paru dans La Recherche de mai 1997.

3. Un bilan carbone sert à comptabiliser les émissions de gaz à effet de serre de toute organisation : entreprises, administration, collectivités, territoire (source Agence de l'environnement et de la maîtrise de l'énergie, Ademe).

4. Le prix Nobel de la paix 2007 a été conjointement décerné au GIEC et à $\mathrm{Al} \mathrm{Gore,}$ ancien vice-président des États-Unis.

\section{RÉSUMÉS}

Avec la création en 1988 du Groupe intergouvernemental d'experts sur l'évolution du climat (GIEC), les changements climatiques émergent sur la scène de la recherche internationale. Un certain nombre de climatologues américains, suédois et anglais donnent l'alerte en créant cette instance. Amy Dahan revient sur cette prise de conscience collective et progressive.

\section{AUTEUR}

\section{AMY DAHAN}

Amy Dahan est directrice de recherche au CNRS, au centre Koyré-Centre de recherche en histoire des sciences et des techniques. 